Journal of Computer Science 8 (4): 579-584, 2012

ISSN 1549-3636

(C) 2012 Science Publications

\title{
Range Detection Multicast Routing Protocol for Mobile Ad-Hoc Networks
}

\author{
${ }^{1}$ Velumani, R. and ${ }^{2} \mathrm{~K}$. Duraiswamy \\ ${ }^{1}$ Department of Computer Science and Engineering, \\ K.S.R. College of Engineering, India \\ ${ }^{2}$ Department Academic, K.S.Rangasamy College of Technology, \\ Tiruchengode-637 215, India
}

\begin{abstract}
Problem statement: Many efforts have been implemented towards the Group membership management on multicast routing in MANETs, but the difficulties associated with Group membership management are still existed. Approach: Maintaining endurance of Group Leader (GL) in multicasting routing has been a great challenge to all the protocol having been invented so far. Results: This study provides the RDMP algorithm for persistency of Group Leader within the group. Nodes perform very active in exchanging the Data and Control packet when they mobile within the transmission range. Range Detection Multicast Protocol (RDMP) focuses on dynamic topology of nodes and uses detection mechanism based on transmission range within the group. Instead of having a separate external node to monitor on Group leadership, a node within the transmission range acts as a Leadership Track Node (LTN), Group Leader manages the group member with the aid provided by LTN and thus control over head shared among them. LTN keeps track of mobility of Group Leader and send alert message when Group Leader moves out of Transmission range and also holds the list of address of all other Group Leader which are adjacent to it. Routing control packet and data packet are regulated with minimum control over head. Conclusion/Recommendations: Our simulation results under increasing group size demonstrate endurance of Group leader for longer period of time, joining delay for multicast group is very low, normalized control over head and minimizing number of hops in maintain average path length.
\end{abstract}

Key words: Group Leader (GL), Leadership Track Node (LTN), Mobile Ad Hoc Networks (MANETs), group membership, Range Detection Multicast Protocol (RDMP)

\section{INTRODUCTION}

Group Communication is more essential in many occasion than one to one communication in the computer communication either it is a wired or wireless communication. Multicast is a one of the examples for best group communication mechanism. The increasing use of Mobile Ad Hoc Networks (MANETs) is prevalent in wireless network system. Mobility of the node in a wireless network is obvious so network topology may change often and out of our prediction. In a MANET, Wireless devices are self configuring in nature and form a network with indigenous topology. As human being on earth often suffering from serious issues like earthquake, tsunami, landslide and battle, Mobile ad hoc networks have become very renowned network system for research in recent years. Research work goes on improving the performance of ad hoc network. Innovations have been implemented in many ways (Xiang et al., 2010; Beraldi and Baldoni, 2003).
Developing a protocol which possesses a reliable and scalable multicast routing is a challenge in the presence of frequent topology and channel dynamics. There are conventional tree based and mesh-based protocols available for MANETs (e.g., LAM (Natsheh and Buragga, 2010), MAODV (Royer and Perkins, 1999) and RSGM (Xiang et al., 2010). Maintaining the tree structures in mobile ad hoc network is very crucial task even though they provide efficient packet delivery. Moreover the tree connection is easy to break and transmission not reliable. To enhance the robustness, the mesh based protocols (e,g., FGMP(Chiang et al., 1998), ODMRP (Lee et al., 2003) are proposed ,They are also not popular due to high degree of overhead as it is difficult to manage group membership, find and maintain multicast paths with constant network topology changes.

\section{MATERIALS AND METHODS}

In this study we design and implement a novel protocol RDMP Range Detection Multicast Routing

Corresponding Author: Velumani, R., Department of Computer Science and Engineering, K.S.R. College of Engineering, India 
Protocol for mobile ad hoc networks for high degree of robustness and persistent enough to be the member head for longer period of time.

Geographic Position System (GPS) Vs transmission range: Even though the state of mobile node is maintained in a Robust and Scalable Geographic Multicast protocol (RSGM) (Xiang et al., 2010), In RSGM several virtual architecture have been constructed as and when group member head changes its position in the network. Virtual tree constructed for packet delivery sometimes lead to unreliable communication. Zone based scheme introduced in this protocol is very confined to certain limit so scalable has become more cumbersome. Number of Group members communicated with group head is considerably low. The moving distance of a mobile node in a zone is very unpredictable. Calculating the average distance of a node moving in a zone changes invariably as network size is increasing. As far as range of transmission of packet is considered, nodes, which are within the transmission range, get the packet without obstacles. Nodes, which are within the interference range, get the packet with a little bit interruption. Either the protocol is source initiative or receiver initiative, during session initiation a node has to broadcast hallow packet for establishing the connection. In this study, we focus on group management, data packet delivery, decreasing the control message over head and ensuring the persistency of group member leader using transmission range detection mechanism.

Range detection multicast protocol: Design approach employed in RDMP is based on range of transmission of packet rather considering zone based group management protocol system. RDMP comes under reactive multicast protocol and constructed by mesh-based architecture. A leader manages the group membership and collects the information about the member nodes in its leadership management. Group leader takes high degree of responsibility to multicast data packets to those entire group member within its range. Unlike RSGM (Xiang et $a l .$, 2010), The group leader is guided and directed by leadership Track node within its range, whereas RSGM requires a separate external source home which would help the group leader. The figure (Fig. 1) shows RDMP's working principles, for presentational convenience, we will first introduce the assumption made and terminologies to be used in the rest of study.

\section{Assumption:}

- A node within a range acts as a Leadership track node

- The Leadership Track Node keep monitoring all other node within the range

- Range limit of about $250 \mathrm{~m}$ is assumed for simulation

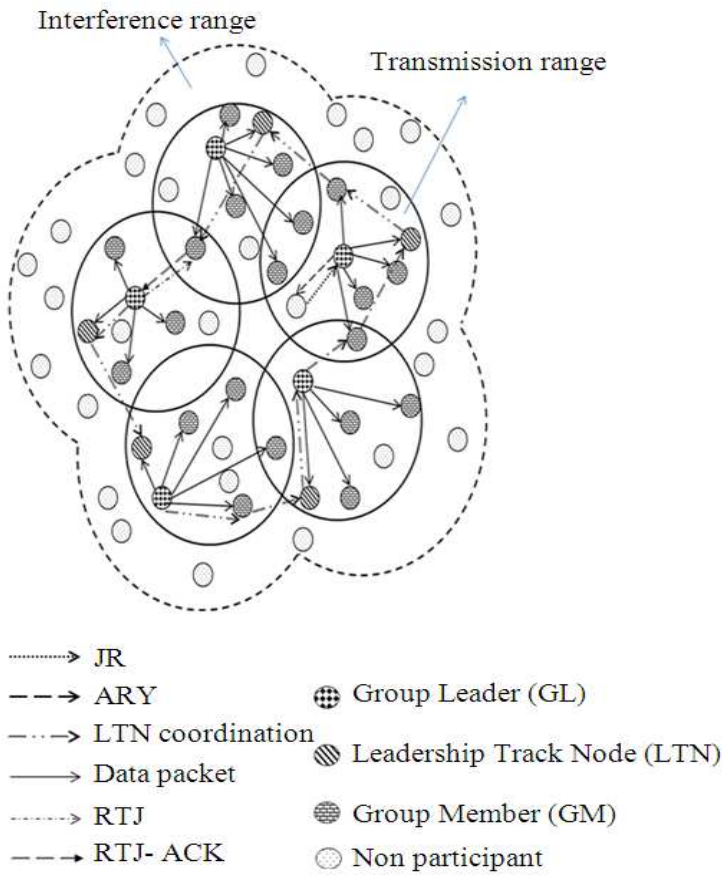

Fig. 1: RDMP's working principle

Terminologies: The minimum number of control messages used in the protocols are RTJ refers to Request to Join made by the node which has maximum of NNR.RTJACK refers to Acknowledgement to RTJ . JR refers to Join Request done by a node which wants to join the group. ARY refers to Acceptance reply done by group leader

Table driven reactive approach: RDMP has become the best example for table driven reactive protocol with a transmission range control mechanism. Each node that takes part in multicast group communication in mobile ad-hoc network is maintaining a table with negligible amount of over head. Nodes in mobile ad hoc network are self configured in organization of data packet delivery and control messages delivery. Data maintained by each node is represented in Table 1 and data maintained by Leadership Track Node is represented in the Table 2 .

Leadership selection: All the nodes within a group want to elect a leadership node under its guidance they want to act upon the group. All the nodes within that group flood the $n$ number of hello packets to each other where $n$ is equal to $\mathrm{n}$ number of nodes in the group. Each node in the group sends the reply ACK (acknowledgement) messages to the entire node reciprocally if it is in the transmission range. As soon as ACK messages received, each node update its table cumulative count value and written into NNR (Number of nodes in the range). 
Table 1: Maintained by individual node

Number of Nodes within a range of Group (NNR)

Number of Group membership in the Group (NGM)

Number of Group Member left in the group (NGL)

Address of Adjacent Group Leader's List (AAGL)

Table 2: Maintained by leadership track node

Number of Nodes within a Range (NNR)

Range Limit of Group Leader (RL)

Address of the group the leader for whom

leadership track acts (AGL)

Address of adjacent Group leader's List (AAGL)

If NNR count of a node is greater than a half of the total number of nodes in the group, then the node is eligible to take part in leadership selection process. For example if $\mathrm{N}$ is 50 in the group, NNR of a node $\mathrm{N}_{\mathrm{i}}$ is 26 then $\mathrm{N}_{\mathrm{i}}$ can take part in the leadership selection process..A node taking part in leadership selection informs all the nodes within range about its NNR value.

A node receiving the NNR value, compare it with its own NNR value, it if it is greater, it will send the RTJ(Request to join) message to all node and update NGM value in its table (number of group membership) on receiving RTJACK(Acknowledgement to RTJ) sent by other node. A node with the highest NGM is announced as the leader of the Group (GL) by leadership Track Node. After leader is selected, Leadership Track Node announces GL's address to all the nodes within the range and updates its own table. Further a node want to join that group may send the JR (Join request) to the group leader and accepted by sending ARY (Acceptance reply) to the node wanted to join and also update its table.

Role of Leadership Track Node (LTN): Among the number of node within the range of multicast group, one acts as a leadership track node.

Purpose of this node is, keep monitoring motion of the Group Leader (GL) within that range. LTN holds the address of the Group leader of its own group as well as all Group leaders' address. Which are adjacent to its Group. Group management is done with help of LTN. Group Leader in a group may to take two kind of Hand over namely, 1.Voluntary Hand over and 2. Hand over due to weak membership strength.

Voluntary handover: Since topology of the group changes, Group Leader of the particular group may about to move beyond the transmission range and get into an interference range, at this juncture interference range is said to be rendezvous point or rendezvous junction. Rendezvous point is a junction where member from different group may get together and hence signal passing through this junction get weakened, Data packet being delivered could not be reached at the destination. When Group leader is about to rendezvous point, leadership track Node inform this matter to group Leader by sending alert message packet. If Group Leader is moving out of the Transmission range deliberately, it would respond the alert message by sending positive acknowledgement with current status maintained by it. On receiving the information about the voluntary retirement of Group Leader, leadership track Node announce the leader selection procedure for that group to select a new Group Leader (GL) with help of the information provided by the Group Leader, which just got voluntary retirement. This type of hand over is called voluntary hand over. This would happen rarely if unavoidable circumstance occurs to the group Leader. If alert message is ignored or reply with negative acknowledgement, Leader Track Node assumes that GL is not moving out of the group.

Handover due to weak membership strength: Instead of flooding the hello packet periodically, It is known that Group Leader has been maintaining the table with necessary information and updating it simultaneously. If a node joining a group or leaving the group, NGM and NGL are updated now and then. Group Leader itself keeps an eye on its own table to make decision on hand over. If NGL increases, automatically NGM decreases. When NGM value comes under very low, Group leader declines its leadership by sending decline message to LTN. LTN will announce Leader selection procedure on receiving decline message from Group Leader. This hand over is called hand over due to weak membership strength.

Algorithm for Group Leader Selection and Monitoring: H-pkt(Hello Packet)

n-(Number of Packet)

$\mathrm{N}-\mathrm{Number}$ of nodes in the Group

$\mathrm{R}_{\mathrm{L}-\text { Range Limit }}$

BEGIN

Flood((H-pkt),n) to N nodes /* Flood

hello packet to all nodes*/

If $\left(\mathrm{N}_{\mathrm{i}}==\mathrm{R}_{\mathrm{L}}\right)$ then

Forward(ACK)by $\mathrm{N}_{\mathrm{i}}$ to all $\mathrm{N}_{\mathrm{i}-1}$ to $\mathrm{N}_{\mathrm{i}-\mathrm{n}}$

$/ *$ If node within range, it will send reply message to all nodes*/

$\mathrm{NNR}==0$

For all $\mathrm{N}_{\mathrm{i}-1}$ to $\mathrm{N}_{\mathrm{i}-\mathrm{n}}$

$\mathrm{NNR}=\mathrm{NNR}++$

/* NNR is updated on

receiving ACK reply*/

Else

$\mathrm{NNR}==0$

For all $\mathrm{N}_{\mathrm{i}-1}$ to $\mathrm{N}_{\mathrm{i}-\mathrm{n}}$

If $\left(\mathrm{NNR}_{\mathrm{i}}>=\mathrm{N} / 2\right)$

Flood(NNR $\mathrm{N}_{\mathrm{i}}$ Value)by $\mathrm{N}_{\mathrm{i}}$ to all

$\mathrm{N}_{\mathrm{i}-1}$ to $\mathrm{N}_{\mathrm{i}-\mathrm{n}} / *$ Inform the NNR 


\author{
value to all nodes in the \\ range*/ \\ If $\left(\mathrm{NNR}\right.$ of $\mathrm{N}_{\mathrm{i}}>\mathrm{NNR}$ of $\left.\mathrm{N}_{\mathrm{i}-1}\right)$ then \\ $\mathrm{NGM}==0$ \\ Flood(RTJ)by $\mathrm{N}_{\mathrm{i}}$ to all $\mathrm{N}_{\mathrm{i}-1}$ to $\mathrm{N}_{\mathrm{i}-\mathrm{n}}$ \\ $/ *$ Request to join the group is sent \\ to all node $* /$ \\ If(RTJACK==1)/*If Positive*/ \\ $\mathrm{NGM}=\mathrm{NGM}++$ \\ /* If positive Ack is received, \\ Number of group membership \\ is updated $* /$ \\ Else \\ Wait to LTN by all $\mathrm{N}_{\mathrm{i}-1}$ to $\mathrm{N}_{\mathrm{i}-\mathrm{n}}$ \\ $/ *$ if negative Ack, Wait for \\ Leadership Track Node*/ \\ For all $\mathrm{N}_{\mathrm{i}}$ to $\mathrm{N}_{\mathrm{i}-\mathrm{n}}$ \\ For all $\mathrm{N}_{\mathrm{j}}$ to $\mathrm{N}_{\mathrm{j}-\mathrm{n}}$ \\ If $\left(\mathrm{NGM}_{\mathrm{i}}>\mathrm{NGM}_{\mathrm{j}}\right)$ \\ $\mathrm{NGM}_{\mathrm{i}}=\mathrm{GL} / *$ A node with \\ highest NGM is called \\ Group Leader by LTN*/ \\ AGL=GL Address by LTN /*LTN \\ Updates GL address*/; \\ Forward(DP-pkt) by GL to all $\mathrm{N}_{\mathrm{i}-1}$ to $\mathrm{N}_{\mathrm{i}-\mathrm{n}}$ \\ If(GL Range $<$ RL) then

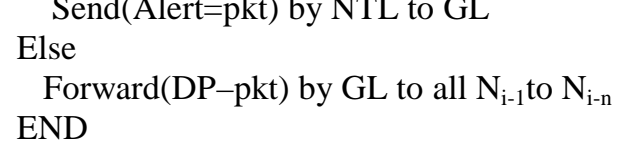

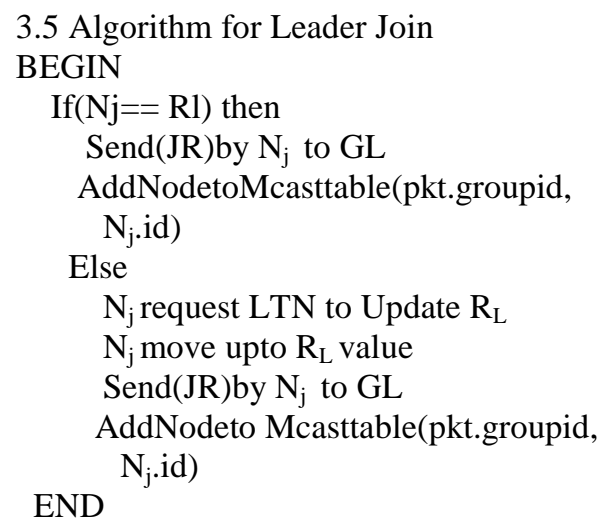

Performance evaluation: We study the performance of RDMP by simulations. We are mainly interested in the protocol's performance on endurance of the group leader for longer period of time without any failure in its group head membership.

Simulation overview: We implemented RDMP with help of NS2 and made use of the idea given in (Li et al., 2000; Abraham et al., 2004) to find the range of the each node. For performance reference, we choose to compare classic mesh-based on demand non Geographic multicast protocol ODMRP (Lee et al., 2003) as it is widely used and considered to be robust protocol SPBM(M. Transier et al., 2004) and stateless RSGM (Xiang et al., 2010) which is designed to improve the scalability of position based multicast protocol. We are mainly interested in the protocol's performance on node's endurance and withstanding capability as leader head for longer period of time and efficiency under the dynamic topology.

Leadership endurance: Once a node in a group selected as the leader for that group, maximum period of time leader keep administrating as ahead is called leadership endurance

Normalized control overhead: Once a node in a group selected as the leader for that group, maximum period of time leader keep administrating as ahead is called leadership endurance

Average path length: The total number of control message transmission divided by the total number of received data packets. RDMP manages to minimize the number of control messages being flooded in the group

Joining delay: The average time intervals between a member joining a group and its first receiving of data packet from that group

\section{RESULTS AND DISCUSSION}

We compare the performance of RDMP with RSGM and SPBM, The performance of the protocol may be impacted by many factors. They are impact of mobility, Impact of node density, impact of Group size, we take the group size is a main factor which determines the exact performance of RDMP. We demonstrate the protocol performance with different group size from minimum 5 members to 250 members. It is well known that group size increases, Density of group also increases. RDMP is very much flexible to increase the group size, thus provide the better scalability in extending the group size. A member selected as group leader using RDMP can remain in this status for longer period of time compared to RSGM and SPBM depicted in the graph shown in Fig. 2a. Number of hops made during data transmission is become low and consistent when group member increases and thus provide very optimum end to end average path length property represented by the graph shown in Fig. 2b. As LTN takes care of movement of GL within the range limit of the group, Join delay is become very low which is shown in the Fig. 2c. 


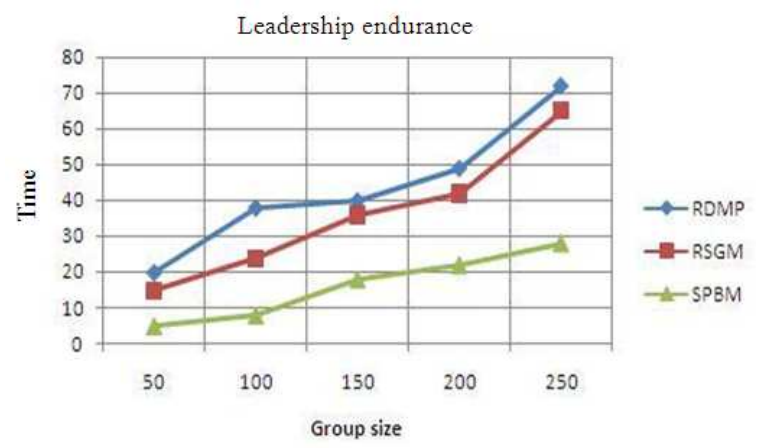

(a)

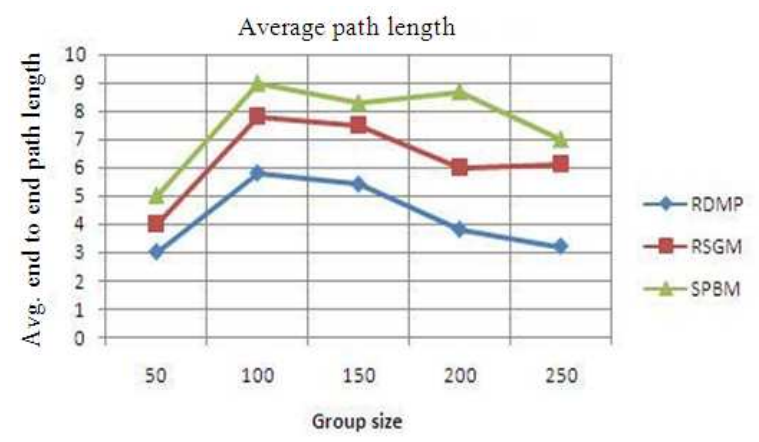

(b)

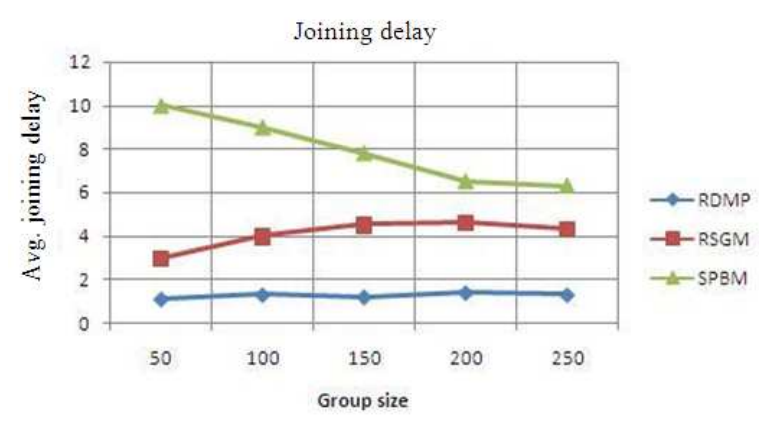

(c)

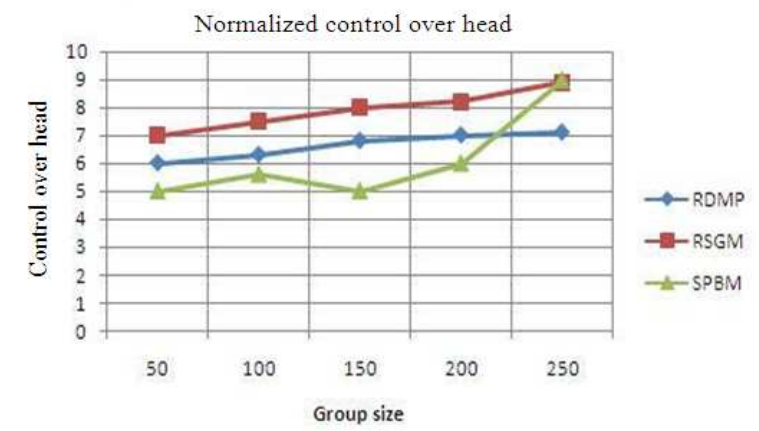

(d)

Fig. 2: (a) Comparison of RSGM and SPBM (b) average path length (c) Join delay (d) monitoring mechanism
As far as control over head is concerned, Number of control messages passed between nodes is very less moreover RDMP is looked after by monitoring mechanism by LTN within the group, the Fig $2 \mathrm{~d}$. shows impact of control messages compared the other two protocols.

\section{CONCLUSION}

In this study, we have designed a persistent Range Detection Multicast Protocol RDMP for MANET. In RDMP, Leadership Track node takes care of all other node in exchanging control messages for efficient group membership management. Both data packets and control messages are transmitted along efficient end-toend tree-like paths without the need of explicitly creating and maintaining a tree structure. A special exclusive hand over mechanism is implemented to elect the best group leader for multicasting. AS RDMP is a table driven reactive multicasting protocol, a member joining the group is carried out only on demand request made by the participant node. To handover the Leadership to other member is carried out by Group Leader by means of voluntary handover mechanism is the special feature of the RDMP.

\section{REFERENCES}

Abraham, I., D. Dolev and D. Malkhi, 2004. LLS: A locality aware location service for mobile Ad Hoc networks. Proceeding of the Joint Workshop on Foundations of Mobile Computing, Sept. 26-Oct. 1, ACM, New York, USA., pp: 75-84. DOI: 10.1145/1022630.1022643

Beraldi, R. and R. Baldoni, 2003. A caching scheme for routing in mobile ad hoc networks and its application to ZRP. IEE Trans. Comput., 52: 10511062. DOI: $10.1109 /$ TC.2003.1223639

Chiang, C.C., M. Gerla and L. Zhang, 1998. Forwarding Group Multicast Protocol (FGMP) for multihop, mobile wireless networks. Cluster Comput., $\quad 1$ : $\quad 187-196 . \quad$ DOI: 10.1023/A:1019037500012

Lee, S.J., W. Su and M. Gerla, 2003. On-Demand Multicast Routing Protocol in Multihop Wireless Mobile Networks. Mobile Networks Applications, 7: 441-453. DOI: 10.1023/A:1020756600187

Li, J., J. Jannotti, D.S.J.D. Couto, D.R Karger and R. Morris, 2000. A scalable location service for geographic Ad Hoc routing. Proceedings of the 6th Annual International Conference on Mobile Computing and Networking, (MCN' 00), ACM, USA., pp: 120-130. DOI: 10.1145/345910.345931 
Natsheh, E. and K. Buragga, 2010. Density based routing algorithm for spare/dense topologies in wireless mobile ad-hoc networks. Am. J. Eng. Applied Sci., 3: 312-319. DOI: 10.3844/ajeassp.2010.312.319

Transier, M., H. Fubler, J. Widmer, M. Mauve and W. Effelsberg, 2004. Scalable position-based multicast for mobile ad-hoc networks. Proceedings of the 1st International Workshop on Broadband Wireless Multimedia: Algorithms, Architectures and Application, (BWMAAA' 04), CiteSeerX, USA.
Royer, E.M. and C.E. Perkins, 1999. Multicast operation of the Ad Hoc on-demand distance vector routing protocol. Proceedings of the 5th Annual ACM/IEEE International Conference on Mobile Computing and Networking, (MCN' 99), ACM, USA., pp: 207-218. DOI: $10.1145 / 313451.313538$

Xiang, X., X. Wang and Y. Yang, 2010. Stateless multicasting in mobile ad Hoc networks. IEEE Trans. Comput., 59: 1076-1090. DOI: 10.1109/TC.2010.102 